\title{
There are No Scalar Lie Fields in Three or More Dimensional Space-Time
}

\section{Klaus Baumann}

Bell Laboratories, Murray Hill, New Jersey 07974, USA, and Max-Planck-Institut für Physik und Astrophysik, D-8000 München, Federal Republic of Germany*

\begin{abstract}
We show that a Lie field structure is incompatible with a scalar relativistic quantum field theory if the dimension of space-time is greater than two. Our main argument is based on the Jacobi identity and the spectrum condition.
\end{abstract}

\section{Introduction}

In 1961 Greenberg [1] suggested to consider fields, for which the commutator in addition to a $C$-number term is linear in the field. Because of this property they were called Lie fields. In the neutral scalar case this would mean

$$
[A(x), A(y)]=\Delta(x, y)+\int d z C(x, y, z) A(z)
$$

where $\Delta$ and $C$ are generalized functions.

Łopuszański [2] remarked that a Lie field theory is soluble in the sense that the Wightman functions are determined by the specification of $\Delta$ and $C$ (see also [3]). Lehmann (see [3]) gave a simple example of a Lie field in two dimensions, namely

$$
A(x)=\varphi(x)+\lambda: \varphi^{2}:(x)
$$

where $\varphi(x)$ is a free field of mass $m$. This is not true in three or more dimensions. Robinson [4] claimed that Lie fields are not possible in more than two dimensions. But his argument was not entirely valid as has been shown by Lowenstein [5]. Lowenstein gave some examples of nonvanishing $C(x, y, z)$ which satisfy the Jacobi identity and are Poincaré invariant. In Section III we shall show that these examples cannot arise from a Wightman theory. Glaser (see [3]) and Greenberg [6] considered more general commutators

$$
\left[A_{i}(x), A_{j}(y)\right]=\Delta_{i j}(x, y)+\sum_{k=1}^{N} \int d z C_{i j k}(x, y, z) A_{k}(z)
$$

with $N$ either finite of infinite and proved that even under these circumstances Lie fields cannot lead to any scattering.

In Section II we list some algebraic properties of Lie fields. Section III contains a reduction of the proof to a simple statement about the Fourier transform of the four-point function. Our main theorem about nonexistence of Lie fields in three or more dimensions will be proved in Section IV. Finally we explain why in two dimensions Lie fields are possible.

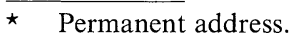




\section{Algebraic Properties of Lie Fields}

For simplicity we restrict ourselves to a single, neutral field $A(x)$, which we assume to fulfill Wightman's axioms [7]. It is no restriction to require $(\Omega, A(x) \Omega) \equiv 0$.

For our purpose it is convenient to rewrite (1.1) in momentum space ([4], [5])

$$
[\tilde{A}(p), \tilde{A}(q)]=\tilde{\Delta}(p, q)+\int d r \tilde{C}(p, q, r) \tilde{A}(-r) \text {. }
$$

Because of Poincaré invariance and locality we have

$$
\begin{aligned}
\tilde{\Delta}(p, q) & =\delta(p+q) \Delta^{\prime}(q) \\
\tilde{C}(p, q, r) & =\delta(p+q+r) C^{\prime}(p, q)
\end{aligned}
$$

where $\Delta^{\prime}$ and $C^{\prime}$ depend only on the Lorentz invariants

$$
\begin{aligned}
\Delta^{\prime}(q) & =\varepsilon\left(q^{0}\right) \cdot \hat{\Delta}\left(q^{2}\right) \\
C^{\prime}(p, q) & =\hat{C}_{i}\left(p^{2}, q^{2},(p+q)^{2}\right)
\end{aligned}
$$

where $i$ is an abbreviation for three indices $\left(i_{p}, i_{q}, i_{p+q}\right)$. The index $i_{p}$ assumes the value,$+ s$, or - according to whether $p$ is a positive time-like, space-like, or a negative time-like vector. (In two dimensions $s$ has to be subdivided in $l$ and $r$ because we can distinguish between space-like vectors lying in the left or right cone!) Therefore the commutation relation looks like

$$
\begin{aligned}
{[\tilde{A}(p), \tilde{A}(q)] } & =\delta(p+q) \Delta^{\prime}(q)+C^{\prime}(p, q) \tilde{A}(p+q) \\
& =\delta(p+q) \varepsilon\left(q^{0}\right) \hat{\Delta}\left(q^{2}\right)+\hat{C}_{i}\left(p^{2}, q^{2},(p+q)^{2}\right) \tilde{A}(p+q) .
\end{aligned}
$$

In addition we have some discrete symmetry properties as

$$
\begin{aligned}
C^{\prime}(p, q) & =-C^{\prime}(q, p) \\
C^{\prime}(p, q)^{*} & =C^{\prime}(-q,-p) .
\end{aligned}
$$

$\Delta^{\prime}(q)$ and $C^{\prime}(p, q)$ are further restricted by the Jacobi identity

$$
[[\tilde{A}(p), \tilde{A}(q)], \tilde{A}(r)]+[[\tilde{A}(r), \tilde{A}(p)], \tilde{A}(q)]+[[\tilde{A}(q), \tilde{A}(r)], \tilde{A}(p)]=0
$$
which implies

$$
\delta(p+q+r)\left\{C^{\prime}(p, q) \Delta^{\prime}(-p-q)+C^{\prime}(r, p) \Delta^{\prime}(-r-p)+C^{\prime}(q, r) \Delta^{\prime}(-q-r)\right\}=0
$$

and

$$
\left\{C^{\prime}(p, q) C^{\prime}(p+q, r)+C^{\prime}(r, p) C^{\prime}(r+p, q)+C^{\prime}(q, r) C^{\prime}(q+r, p)\right\} \tilde{A}(p+q+r)=0 .
$$

\section{Reduction of the Proof}

In Section IV we shall show that the spectrum condition and the Jacobi identity imply that in three or more dimensions

$$
(\Omega, \tilde{A}(s) \tilde{A}(p) \tilde{A}(q) \tilde{A}(r) \Omega) \equiv 0
$$

for all space-like momenta $p$ and $q$.

We want to explain here why $C^{\prime}(p, q) \equiv 0$ is a consequence of Eq. (3.1). 
Lemma. A scalar relativistic field $A(x)$ for which $(\Omega, \tilde{A}(s) \tilde{A}(p) \tilde{A}(q) \tilde{A}(r) \Omega) \equiv 0$ if $p^{2}<0$ and $q^{2}<0$ is a generalized free field [1], i.e.

$[A(x), A(y)]=\Delta(y-x)$.

Proof. $(\Omega, \tilde{A}(s) \tilde{A}(p) \tilde{A}(q) \tilde{A}(r) \Omega) \equiv 0$ for all $p^{2}<0$ and $q^{2}<0$ yields immediately $\tilde{A}(q) \tilde{A}(r) \Omega \equiv 0$ for all $q^{2}<0$.

The Jost-Lehmann-Dyson representation then implies that

$$
\begin{aligned}
{[A(x), A(y)] \Omega } & =(\Omega,[A(x), A(y)] \Omega) \cdot \Omega \\
& =\Delta(y-x) \cdot \Omega .
\end{aligned}
$$

(For a proof see Greenberg [8] and Robinson [9].) The Reeh-Schlieder theorem [7] proves our lemma.

Remark. The examples for nontrivial $C^{\prime}(p, q)$ given by Lowenstein [5] are such that

$$
[\tilde{A}(p), \tilde{A}(q)] \equiv 0 \quad \text { if } \quad p^{2}<0
$$

and therefore cannot be used to construct relativistic scalar Lie fields.

\section{The Main Result}

In the following we restrict ourselves to three of more dimensional space-time.

Theorem. If $A(x)$ is a scalar, relativistic Lie field then $(\Omega, \tilde{A}(s) \tilde{A}(p) \tilde{A}(q) \tilde{A}(r) \Omega) \equiv 0$ for all space-like momenta $p$ and $q$.

Proof.

a) Jacobi identity:

We want to use the Jacobi identity (2.7) for the special case $p^{2}<0, q^{2}<0$, and $r \in \bar{V}_{+}$

$$
\begin{aligned}
0= & (\Omega, \tilde{A}(s)\{[[\tilde{A}(p), \tilde{A}(q)] \tilde{A}(r)]+[[\tilde{A}(r), \tilde{A}(p)] \tilde{A}(q)] \\
& +[[\tilde{A}(q), \tilde{A}(r)] \tilde{A}(p)]\} \Omega) \\
= & \delta(p+q+r+s) \Delta_{+}(p+q+r) \\
& \cdot\left\{C^{\prime}(p, q) C^{\prime}(p+q, r)+C^{\prime}(r, p) C^{\prime}(r+p, q)+C^{\prime}(q, r) C^{\prime}(q+r, p)\right\} \\
= & \delta(p+q+r+s) \Delta_{+}(p+q+r) \\
& \cdot\left\{\tilde{C}\left(p^{2}, q^{2},(p+q)^{2}\right) \hat{C}\left((p+q)^{2}, r^{2},(p+q+r)^{2}\right)\right. \\
& +\hat{C}\left(r^{2}, p^{2},(r+p)^{2}\right) \hat{C}\left((r+p)^{2}, q^{2},(p+q+r)^{2}\right) \\
& \left.+\hat{C}\left(q^{2}, r^{2},(q+r)^{2}\right) \hat{C}\left((q+r)^{2}, p^{2},(p+q+r)^{2}\right)\right\} .
\end{aligned}
$$

Here $\Delta_{+}(p+q+r)$ means the positive frequency part of $\Delta^{\prime}(p+q+r)$. 
b) Spectrum condition:

The second and the third term in (4.1) are restricted by the spectrum condition $(\Omega, \tilde{A}(s)[[\tilde{A}(r), \tilde{A}(p)] \tilde{A}(q)] \Omega)$

$=C^{\prime}(r, p)(\Omega, \tilde{A}(s)[\tilde{A}(r+p), \tilde{A}(q)] \Omega)=0 \quad$ if $\quad(r+p)^{2}<0$

$(\Omega, \tilde{A}(s)[[\tilde{A}(q), \tilde{A}(r)] \tilde{A}(p)] \Omega)$

$=C^{\prime}(q, r)(\Omega, \tilde{A}(s)[\tilde{A}(q+r), \tilde{A}(p)] \Omega)=0 \quad$ if $\quad(q+r)^{2}<0$.

c) The geometrically allowed region:

Following Robinson [4] we introduce the variables

$$
\begin{aligned}
& u=p^{2}, \quad v=q^{2}, \quad u^{\prime}=r^{2}, \quad v^{\prime}=(p+q+r)^{2} \\
& w=(p+q)^{2}, \quad w^{\prime}=(q+r)^{2}, \quad t=(p+r)^{2}=u+v+u^{\prime}+v^{\prime}-w-w^{\prime} .
\end{aligned}
$$

Now we consider Eq. (4.1) for fixed $u, v<0$ and $u^{\prime}, v^{\prime} \geqq 0$. Then $w$ and $w^{\prime}$ can only vary in a certain region of the $w, w^{\prime}$-plane - the geometrically allowed region. We refer to Lowenstein [5] for a detailed analysis.

For our special choice of $u, v, u^{\prime}$, and $v^{\prime}$ the geometrically allowed region can be described quite easily. The $w$-axis is divided by four critical points

$$
\begin{aligned}
& w_{1,2}=u+v \mp 2 \sqrt{u v}, \quad w_{3,4}=u^{\prime}+v^{\prime} \mp 2 \sqrt{u^{\prime} v^{\prime}} \\
& w_{1}<w_{2} \leqq 0 \leqq w_{3} \leqq w_{4} .
\end{aligned}
$$

Define

$$
\begin{aligned}
w_{ \pm}^{\prime}(w)= & v+u^{\prime}-(2 w)^{-1}(w-u+v)\left(w+u^{\prime}-v^{\prime}\right) \\
& \pm(2 w)^{-1} \sqrt{\left(w-w_{1}\right)\left(w-w_{2}\right)\left(w-w_{3}\right)\left(w-w_{4}\right)} .
\end{aligned}
$$

The geometrically allowed region has the following form:

$\alpha) w \geqq w_{4}$

$w^{\prime}$ lies between $w_{ \pm}^{\prime}(w)$

в) $w_{3}<w<w_{4}$

geometrically impossible

r) $0 \leqq w \leqq w_{3}$

$w^{\prime}$ lies between $w_{ \pm}^{\prime}(w)$

d) $w_{2} \leqq w \leqq 0$

$w^{\prime}$ lies outside $w_{ \pm}^{\prime}(w)$

ह) $w_{1} \leqq w \leqq w_{2}$

every value of $w^{\prime}$ is possible

ל) $w \leqq w_{1}$

$w^{\prime}$ lies outside $w_{+}^{\prime}(w)$.

d) Implication of Jacobi identity and spectrum condition:

In the new variables Eq. (4.1) gives

$$
\begin{aligned}
& \delta(p+q+r+s) \hat{\Delta}\left(v^{\prime}\right)\left\{\hat{C}(u, v, w) \hat{C}\left(w, u^{\prime}, v^{\prime}\right)\right. \\
& \left.+\hat{C}\left(u^{\prime}, u, t\right) \hat{C}\left(t, v, v^{\prime}\right)+\hat{C}\left(v, u^{\prime}, w^{\prime}\right) \hat{C}\left(w^{\prime}, u, v^{\prime}\right)\right\}=0 .
\end{aligned}
$$


Fig. 1. Sketch of the geometrically allowed region for $u<v<0<u^{\prime}<v^{\prime}$

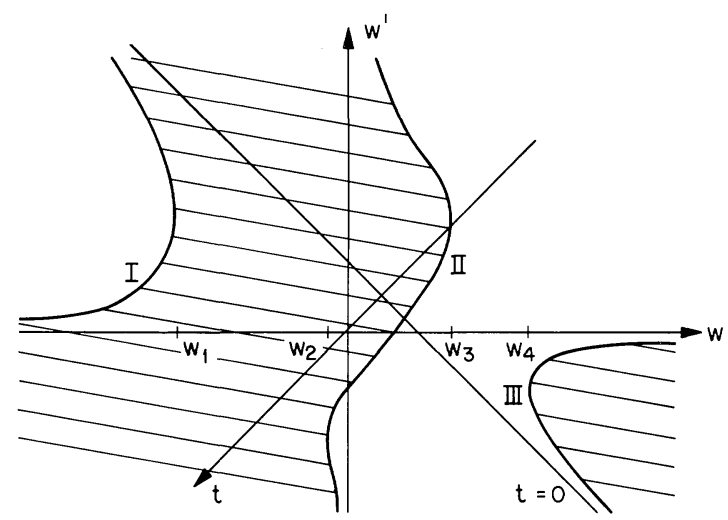

For fixed $u, v, u^{\prime}$, and $v^{\prime}$ (we neglect the overall $\delta$-function) we get

$$
\hat{\Delta}\left(v^{\prime}\right)\left\{f(w)+g(t)+h\left(w^{\prime}\right)\right\}=0
$$

where $w, w^{\prime}$ and $t=u+v+u^{\prime}+v^{\prime}-w-w^{\prime}$ can vary in the geometrically allowed region. The spectrum condition (4.2) reads

$$
\begin{array}{cll}
\hat{\Delta}\left(v^{\prime}\right) g(t)=0 & \text { for } & t<0 \\
\hat{\Delta}\left(v^{\prime}\right) h\left(w^{\prime}\right)=0 & \text { for } & w^{\prime}<0 .
\end{array}
$$

Differentiating (4.7) with respect to $w$ and $w^{\prime}$ gives $\hat{\Delta}\left(v^{\prime}\right) g^{\prime \prime}(t) \equiv 0$ and (4.8) implies

$$
\hat{\Delta}\left(v^{\prime}\right) g(t) \equiv 0 \text {. }
$$

In a similar way we get

$$
\hat{\Delta}\left(v^{\prime}\right) h\left(w^{\prime}\right) \equiv 0
$$

and finally

$$
\hat{\Delta}\left(v^{\prime}\right) f(w) \equiv 0 \text {. }
$$

But this means

$$
\begin{aligned}
-(\Omega, \hat{A}(s)[[\tilde{A}(q), \tilde{A}(r)] \tilde{A}(p)] \Omega) & =(\Omega, \tilde{A}(s) \tilde{A}(p)[\tilde{A}(q) \tilde{A}(r)] \Omega) \\
& =(\Omega, \tilde{A}(s) \tilde{A}(p) \tilde{A}(q) \tilde{A}(r) \Omega)=0
\end{aligned}
$$

for all space-like momenta $p$ and $q$ and all momenta $r \in \bar{V}_{+}$. This proves our theorem.

We see that the Jacobi-identity and the spectrum condition together are too restrictive to allow Lie fields in three or more dimensions.

\section{Remark about Two Dimensional Lie Fields}

We know that there are Lie fields in two dimensions. The reason for this is purely geometrical. For fixed $p^{2}, q^{2}<0$ and $r^{2},(p+q+r)^{2} \geqq 0$ the geometrically allowed region consists only of the lines I, II and III and has no open interior region 
whatever. This means $w=(p+q)^{2}$ determines $w^{\prime}=(q+r)^{2}$ and $t=(p+r)^{2}$. It is perhaps interesting what relative configurations of the four hyperbolas the different lines correspond to. We fix $r^{2}=$ const. in the forward cone and $p^{2}=$ const. in the left space-like cone. Then we have for line $I$ :

$$
\begin{aligned}
q^{2} & =\text { const. in the left space-like cone } \\
(p+q+r)^{2} & =\text { const. in the forward cone }
\end{aligned}
$$

for line II:

$$
\begin{aligned}
q^{2} & =\text { const. in the right space-like cone } \\
(p+q+r)^{2} & =\text { const. in the forward cone }
\end{aligned}
$$

for line III:

$$
q^{2}=\text { const. in the right space-like cone }
$$

$$
(p+q+r)^{2}=\text { const. in the backward cone. }
$$

Whereas in three or more dimensions we have two configurations, namely $r^{2}=$ const. and $(p+q+r)^{2}=$ const. lie either in the same or in the opposite time-like cones.

Acknowledgement. I want to thank Drs. J. R. Klauder, H. Narnhofer, and E. Seiler for many helpful discussions and suggestions.

\section{References}

1. Greenberg, O. W.: Ann. Phys. 16, 158 (1961)

2. Łopuszański, J.T.: Phys. Lett. 8, 85 (1964)

3. Wightman, A.S.: 1964 Cargèse Lectures in Theoretical Physics (ed. M.Lévy), pp. 191-196. New York: Gordon and Breach 1967

4. Robinson, D. W.: Phys. Lett. 9, 189 (1964)

5. Lowenstein, J.H.: Commun. math. Phys. 6, 49 (1967)

6. Greenberg, O. W.: Commun. math. Phys. 9, 13 (1968)

7. Streater, R.F., Wightman, A.S.: PCT, Spin and Statistics and All That. New York: Benjamin 1964 1964

Jost, R.: The General Theory of Quantized Fields. Providence: AMS 1965

8. Greenberg, O.W.: J. Math. Phys. 3, 859 (1962)

9. Robinson, D. W.: Helv. Phys. Acta 35, 403 (1962)

Communicated by A. S. Wightman

(Received June 24, 1975) 